\title{
Textes sur la méthode et l'organisation de l'intervention sociale
}

\section{Florence Kelley}

Translator. Andrea Cavazzini

\section{OpenEdition}

\section{Journals}

Electronic version

URL: https://journals.openedition.org/grm/2978

DOI: $10.4000 / \mathrm{grm} .2978$

ISSN: $1775-3902$

\section{Publisher}

Groupe de Recherches Matérialistes

\section{Electronic reference}

Florence Kelley, "Textes sur la méthode et l'organisation de l'intervention sociale ", Cahiers du GRM [Online], 17 | 2020, Online since 02 January 2021, connection on 30 September 2021. URL: http:// journals.openedition.org/grm/2978; DOI: https://doi.org/10.4000/grm.2978

This text was automatically generated on 30 September 2021.

(c) GRM - Association 


\title{
Textes sur la méthode et l'organisation de l'intervention sociale
}

\author{
Florence Kelley
}

Translation : Andrea Cavazzini

\section{Objectifs et principes de la Ligue des consommateurs (in American Journal of Sociology, 3, 1899, p. 289-304)}

1 Les principes fondamentaux de la Ligue des consommateurs sont peu nombreux et simples. Ils sont à la fois économiques et moraux. Le premier principe de notre Ligue est l'universalité : elle reconnaît que dans une communauté civilisée chaque individu est un consommateur. Depuis le berceau - qui peut être en bois ou en métal, avec ou sans bascule - jusqu'au cercueil - auquel on peut toujours préférer une urne - à travers toute notre vie nous choisissons, ou plus précisément des choix nous sont imposés, en fonction de la disponibilité d'argent. Du jeune vendeur de journaux qui alimente l'industrie des cigarettes et du chewing-gum, et qui est lui-même alimenté par notre incapacité à lui préférer un père de famille manchot lorsque nous achetons notre journal, jusqu'au patron sensible et consciencieux de la Kelmscott Press, nous tous nous faisons des choix, chaque jour et chaque instant, concernant l'usage des moyens dont nous disposons. En faisant cela, nous contribuons à déterminer, bien que d'une manière inconsciente, la manière dont nos semblables vivront pendant le temps requis pour produire ce que nous achetons.

2 Rares sont parmi nous ceux qui peuvent dépenser des sommes importantes en actes de charité : donner une dîme est peut-être un peu exorbitant par rapport aux habitudes actuelles. Mais, quelle que soit la portée de nos dons, ils sont moins décisifs pour le bonheur ou le malheur de nos frères que nos dépenses habituelles. Car un homme est dans une large mesure ce que son travail fait de lui: un artiste, un artisan, un 
travailleur manuel, une bête de somme, une victime des contremaîtres, ou, à peine moins digne de pitié, un contremaitre. Toutes ces catégories de travailleurs, parmi de nombreuses autres, existent pour fournir la demande que nous incarnons, nous et nos concitoyens.

3 Ceux d'entre nous qui jouissent du privilège de voter peuvent contribuer, une ou deux fois par an, à déterminer la manière dont les tarifs, la monnaie ou les impôts locaux devraient être adaptés à nos industries. Mais chacun d'entre nous détermine, tout le temps, à travers ses dépenses, la survie de telle ou telle industrie, et les conditions de cette survie.

4 Pour parler simplement, le but de la Ligue nationale des consommateurs est de moraliser cette décision, de recueillir et de rendre disponibles des informations qui pourront rendre tout un chacun capable de décider à la lumière d'une connaissance éclairée et de faire appel à sa conscience, pour faire en sorte que la décision prise soit une juste décision.

5 La Ligue des consommateurs, ainsi, agit, d'un côté, sur la base de l'idée que le consommateur détermine en dernière instance toute production, car tout article doit cesser d'être produit si tous les consommateurs cessent de l'acheter, tel le mobilier en crinière de cheval si répandu au début du siècle, et qui maintenant n'est pratiquement plus fabriqué; tandis que, d'un autre côté, n'importe quelle marchandise, en dépit de l'attente à la vie et à la santé humaines que portent ses conditions de productions, ou du risque qu'elles impliquent, continue à circuler sur le marché tant qu'une demande effective persiste: par exemple, la nitroglycérine, le phosphore et toute sorte de produits pour le travail dans les mines.

6 Toutefois, alors même que la grande masse des consommateurs détermine, d'une manière assez générale et sur la longue période, ce qui finira par être produit, le consommateur individuel ne dispose pas actuellement, à cause de son manque d'organisation et de connaissances techniques, des moyens adéquats pour faire connaître ses désirs et pour faire de ses demandes des demandes effectives. La vérité de ce principe est illustrée par une multitude d'exemples tirés de l'expérience de chacun. Un cas affligeant de consommation non-effective peut être indiqué au sein de la colonie des immigrés italiens dans toutes nos grandes villes. Ces communautés entretiennent au moins un commerce pour l'importation de pâtes, spaghetti, saucisson de Bologne ou autres marques de charcuterie, huile d'olive, Chianti, fromages et noix italiens... Ces marchandises sont toutes excessivement coûteuses, à cause des tarifs et des impôts pour l'importation et l'exportation, mais ces immigrés ont l'habitude de les utiliser, et préfèrent une quantité plus petite de ces sortes de nourriture à une plus grande quantité des marchandises moins chères et plus accessibles dont ils sont entourés.

7 Le pitoyable résultat est que les marchands achètent la plus petite quantité possible du vrai produit italien afin de la mélanger avec des produits adultérants américains. L'exemple le plus flagrant de cela est probablement la vente au détail de l'huile d'olive italienne, dont aucune quantité réellement pure ne circule sur le marché. Ce que les immigrés italiens obtiennent est en réalité la marque italienne familière, un paquet bien connu dont les contenus ont plus ou moins le goût auquel ils étaient habitués en Italie.

8 Ils savent ce que sont réellement les ingrédients qu'ils consomment aussi peu que nous savons ce que contient le soi-disant sirop d'érable, ou les prétendus beurre ou miel que nous étalons sur nos gâteaux chauds dans un salon en ville. La demande des Italiens 
vivant aux États-Unis concernant des produits italiens, bien que vaste, persistante et soutenue par des lourds sacrifices de la part des acheteurs, n'est pas une demande effective, car les immigrés ne disposent pas de la connaissance, ni de l'organisation, requises pour la mettre en pratique. Que la seule connaissance dépourvue d'organisation soit insuffisante pour créer une demande effective est clairement montré par l'expérience d'une cliente consciencieuse que j'ai rencontrée à Chicago.

Profondément touchée par un appel éloquent en faveur des victimes des contremaîtres et de leurs souffrances, elle a décidé de soulager sa conscience en achetant uniquement des marchandises produites dans des usines. Elle commença sa recherche de telles marchandises dans la grande boutique du département où elle avait toujours acheté à ses enfants leurs outils pour l'école. Le vendeur la rassura en disant que "nos marchandises sont produites dans notre propre usine, nous ne traitons pas des produits venant des ateliers de misère ». L'acheteuse, qui est une personne intelligente et instruite, demanda une déclaration écrite, signée par un membre de l'entreprise, et que cette déclaration lui soit envoyée à la maison avec les achats. Rien ne fut jamais envoyé, malgré la fidélité de la cliente, que l'entreprise avait tout intérêt à satisfaire et à ménager dans les limites du possible.

Elle fit plusieurs tentatives identiques auprès de nombreuses boutiques, jusqu'à ce qu'elle comprenne qu'elle ne pouvait pas soulager sa conscience seule et sans aide. Sa situation critique montre bien ce qui arrive aux acheteurs isolés, éclairés mais non organisés, et de ce fait inefficaces.

11 L'acheteur qui a la capacité et la volonté de payer pour obtenir ce que le marché peut fournir de mieux peut certes croire qu'il est à l'abri des malheurs des acheteurs de marchandises ready-made, car il n'achète ses vêtements que chez le tailleur et paie un prix très élevé pour avoir la certitude qu'ils sont produits dans des conditions qui le protègent contre les maladies, et permettent au marchand de payer l'ouvrier tailleur un prix adéquat à son labeur.

12 Mais cet acheteur n'est pas vraiment mieux loti que la colonie des Italiens ou que la femme instruite mais inefficace qui recherche des marchandises rigoureusement readymade. Par exemple, quand j'étais inspecteur d'usine dans l'Illinois, j'étais en train un jour de chercher un fabriquant de cigares dont on disait qu'il avait des cas de variole dans sa famille, pendant la terrible épidémie de 1894. Je suis tombée par un pur hasard sur un tailleur qui venait d'emménager dans la maison que l'on croyait contaminée, et qui n'était toujours pas enregistré auprès du bureau local de santé ni de mon département. Dans cette boutique de tailleur, qui était aussi son domicile, il y avait un cas de variole. Dans la même boutique se trouvait aussi un très bon manteau, du type que des messieurs distingués payaient cette année-là entre 60 et 75 dollars. Dans le col du manteau, un bandeau indiquait le nom du tailleur le plus important de Helena, dans le Montana. Or, ce marchand tailleur gardait, dans sa vitrine, des vêtements d'une excellente qualité, parmi lesquels le client avait choisi son manteau.

13 Le tailleur avait pris ses mesures et les avait envoyées par le télégraphe, avec le numéro de l'échantillon, au grand magasin de Chicago dont il était un affilié. Le magasin avait travaillé sur le manteau et l'avait envoyé au tailleur, et c'est chez celui-ci, dans sa chambre de malade à l'intérieur d'un bâtiment infect, qu'il avait été retrouvé. Faute de l'heureuse coöncidence qui a fait que nous avons trouvé le tailleur en recherchant quelqu'un d'autre, le client malchanceux habitant Helena, dans le Montana, aurait sans doute acheté des germes de variole avec son vêtement si cher payé. 
14 La position de cet acheteur ne différait pas d'une manière essentielle de celle des immigrés italiens. Tout comme ces derniers, il était en train de payer une somme d'argent assez importante pour obtenir des biens sûrs; mais, encore une fois tout comme eux, il ne disposait pas des connaissances et de l'organisation qui auraient pu rendre sa demande effective. Outre la croyance infondée selon laquelle un coût élevé de la production implique des conditions hygiéniques adéquates, l'acheteur de vêtements chers se rassure habituellement en supposant que les ouvriers tailleurs qui produisent de tels vêtements reçoivent une partie consistante du prix sous la forme d'un salaire adéquat.

15 Certes, il est vrai que la Fraternité des Tailleurs peut imposer, grâce à une forte organisation, de meilleurs salaires que les travailleurs dans les secteurs du readymade; toutefois, il est vrai aussi que le tailleur qui nous occupe, ainsi que de nombreux autres dont j'ai eu connaissance, était poussé par son extrême pauvreté à cacher la circonstance épouvantable de la circulation de la variole dans sa famille, car il redoutait la perte de quelques jours ou de quelques semaines de travail.

16 Ainsi, le prix élevé de son manteau ne garantissait même pas au client habitant Helena, dans le Montana, la simple bonne conscience concernant le montant des salaires. La question est parfois posée de savoir si, malgré les cas particuliers susmentionnés, et les maux qu'ils incarnent de manière typique, les lois ne protègent pas l'acheteur, et si le producteur ne consacre pas toutes ses énergies pour satisfaire les vœux des consommateurs, si bien qu'une autre organisation dans cette époque si riche en organisations ne serait pas superflue. Cette question en contient deux différentes, qui doivent obtenir deux réponses séparées.

17 Premièrement, en ce qui concerne le producteur et son effort d'être à la hauteur des vœux du consommateur : certes, il est vrai que chaque entrepreneur des manufactures étudie le marché, qu'il est forcé, s'il veut réussir dans ses affaires, de calculer, de déduire, de deviner, à partir des actions de l'acheteur de l'année dernière, de la veille et d'aujourd'hui, les actions de l'acheteur de demain et de l'année prochaine. La faillite d'une partie congrue d'industriels manufacturiers montre la difficulté d'un tel travail de déduction. Les crises récurrentes montrent que la difficulté est parfois insurmontable pour le corps total de la manufacture tout entière. Des industriels qui réussissent correspondent aux désirs de vastes masses d'acheteurs; mais la correspondance est loin d'être toujours assez étroite pour être satisfaisante. Combien de fois constatons-nous que nos bouquins reliés à la manière ready-made, et nos chaussures ou d'autres vêtements, ne sont pas tels que nous les souhaiterions? Le pain de boulangerie est un exemple classique de nourriture ready-made visant à satisfaire l'acheteur "moyen ", et qui ne correspond au goût de qui que ce soit. Les difficultés du travailleur des manufactures sont fortement intensifiées par l'incompétence extraordinaire de l'acheteur " moyen » en ce qui concerne l'évaluation selon le mérite. Quelle ménagère pourrait déceler, seule et sans aide, les produits chimiques nuisibles dans son lait, son pain, sa viande, ses produits pour la maison? Quelle jeune fille choisissant de la soie pour ses ornements sait que le taffetas bouilli dans l'huile est plus durable que la soie ordinaire pour un prix deux fois supérieur, ou connaît les causes de cela? Et nous achetons nos roues sur la base de la réputation du fabriquant, sans rien connaître aux qualités de la gomme, de l'acier, du laiton, du bois et du cuir utilisés pour les fabriquer. 
18 Dans certaines grandes industries modernes, des manières pour tester le produit ont été inventées; si bien que les navires de guerre, les locomotives, les ponts ferroviaires et les installations électriques peuvent tous être testés avant que l'addition soit payée. Cependant, en ce qui concerne la grande masse des produits de l'industrie contemporaine, rien n'a été inventé qui corresponde à ces tests. C'est particulièrement vrai pour tous ces secteurs de la manufacture qui étaient autrefois le fait des femmes au foyer, et qui se sont déplacés maintenant vers les ateliers et les fabriques. S'agissant de ces produits, les clients ne peuvent compter que sur leur habileté individuelle d'acheteurs. L'ancienne règle caveat emptor est ici portée jusqu'à ses conséquences les plus extrêmes. Et c'est pourquoi les producteurs souffrent tellement à cause du manque d'intelligence qui caractérise les consommateurs qu'ils sont en train de construire des musées dont le seul but est d'éduquer les acheteurs, et dont le nouveau Musée du commerce de Philadelphia peut être considéré comme un prototype prometteur.

Toutefois, tandis que les industriels manufacturiers les plus éclairés et progressistes abordent leurs problèmes par le biais de l'éducation, la grande masse des producteurs ont recours depuis longtemps au dispositif bien plus simple de la publicité, qui ne peut guère revendiquer une grande valeur éducative, car, loin d'être consacrée à éduquer et à instruire, elle ne vise qu'à stimuler, persuader, inciter, séduire, et faire en sorte que les indifférents commencent à acheter. La plupart des publicités existantes, dont le type idéal pourrait être la publicité des médicaments brevetés, font directement appel à l'ignorance des acheteurs. Elles visent presque exclusivement la cupidité du public, et partant mettent en avant les bas prix comme leur caractéristique principale. Or, la Ligue des Consommateurs n'a rien contre la réduction des prix obtenue en introduisant des machines avec trois, quatre ou dix aiguilles, alimentées par des dynamos et utilisées pour coudre des produits issus de ciseaux électriques. Mais elle trouve beaucoup à redire lorsqu'il s'agit de réductions de prix obtenues par le travail d'enfants qui activent des machines alimentées par l'énergie de leurs jambes, enfermés dans les cuisines de grands immeubles et mis en concurrence avec les appareils électriques. La Ligue essaie ainsi de fournir des informations par lesquelles l'acheteur potentiel peut vérifier la véridicité des affirmations du producteur lorsque celui-ci assure vouloir se conformer aux désirs du public. Au producteur, la Ligue propose ce dont il a plus besoin que de n'importe quelle autre condition de réussite - une population de clients stable et fidélisée.

21 En Angleterre, où le mouvement coopératif a lentement grandi jusqu'à atteindre une taille gigantesque, les clients ont pu, en unifiant leurs intérêts, employer des experts capables de prescrire à l'avance les conditions de la production ainsi que les prix et la qualité, en obtenant en retour des biens produits pour la demande persistante et stable qu'ils représentent aux yeux de producteurs déjà conscient des souhaits de cette partie de leur public d'acheteurs. Dans notre pays, faute d'une telle organisation, l'offre et la demande sont laissées à leur propre régulation automatique, ce qui finit par ruiner de nombreux marchands et producteurs qui essayent sans succès de deviner les souhaits du public ou qui échouent à l'interpeller par leurs offres s'adressant à sa cupidité et à son ignorance supposées, par nous impliquer tous dans la consommation d'immenses quantités de marchandises avariées et défectueuses produites par des tentatives de se rapprocher des souhaits d'une population d'acheteurs non éclairée et non organisée, et finalement par faire descendre sous le niveau de la simple survie les salaires de la partie la plus faible des employés qui produisent et distribuent de telles marchandises. 
La Ligue des Consommateurs reconnaît que ces déductions, ces inférences, ces suppositions à l'égard des souhaits du consommateur, menées d'une manière aveugle à partir des comportements passés de ce dernier, si elles sont universellement répandues dans ce pays, ne sont pas pour autant inévitables au nom d'une quelconque loi naturelle ou sociale. Toute législation sur le travail des usines suppose la reconnaissance du fait que les relations humaines de l'offre et de la demande sont susceptibles d'être modifiées dans un sens bénéfique; le mouvement coopératif témoigne aussi de ce même état de choses ; la Ligue des Consommateurs, dernier acteur en date dans ce domaine, vise à fournir une démonstration ultérieure de cette vérité. En ce qui concerne la deuxième partie de la question de savoir si le consommateur n'est pas réellement protégé par les lois et éclairé par les informations officielles que de telles lois impliquent - à cette question la réponse est multiforme, malgré les exemples individuels déjà mentionnés d'une absence de pouvoir de l'acheteur isolé.

22 L'une des considérations les plus importantes est que la législation n'est nullement uniforme entre les États; si bien que l'homme probe du Massachusetts, vivant sous le meilleur code du travail que ce pays ait connu, dont le respect est assuré par les inspecteurs d'usine les plus attentifs et compétents, risque autant que l'acheteur du Montana qui fait ses courses dans les boutiques de Chicago d'acheter des vêtements produits dans les atelier infects dominés par le sweat system, qui bat son plein et n'a de cesse de se développer chaque jour en intensité et en extension dans la ville de New York. Car suivant la Constitution des États-Unis, aucun État ne peut interdire l'importation de marchandises produites dans un autre État, quelle que soit l'infériorité des critères imposés aux conditions du travail manufacturier dans l'État producteur par rapport à ceux de l'État importateur. La lutte pour une législation uniforme protégeant tous les consommateurs semble justifier, faute d'autre justification, le travail de la Ligue Nationale des Consommateurs.

23 Ce n'est pas tout. Tant que les manufactures dépensent des millions de dollars afin de séduire et de persuader des acheteurs, les États et les villes dépensent des centaines de milliers de dollars afin de fournir au public des informations à propos des conditions industrielles, des adultérations des produits alimentaires, et d'autres intérêts des acheteurs. Le Département du Travail à Washington, les bureaux des États pour les statistiques sur le travail, les inspecteurs des usines dans les États, les conseils municipaux de la santé publient tous, une fois ou deux par an - certains le font chaque mois et même chaque semaine - des informations visant à éclairer et à instruire le public. Mais une toute petite partie de ces informations ont servi jusqu'ici les intérêts de l'acheteur individuel. Après avoir lu les rapports de toutes ces instances publiques, non seulement le risque n'en est pas moindre qu'avant d'acheter du glucose à la place du sucre, de l'acide acétique à la place du vinaigre, du papier dans les semelles de mes chaussures. Et je risque toujours autant d'acheter de la variole, de la scarlatine, de la rougeole, des infections des yeux et une douzaine de maladies de la peau dans mes nouveaux vêtements. Car aucune de ces instances ne publie la liste des tailleurs auxquels les marchands de vêtements confient la préparation de leurs marchandises. Et aucune d'entre elles ne peut donner des informations sur la base desquelles des adultérations de la nourriture peuvent être repérées avec succès dans la cuisine des particuliers.

24 Il est donc urgent qu'une société privée enquête sur certains secteurs industriels et dresse une liste des meilleurs établissements dans ledit secteur, à savoir ceux qui 
garantissent que le produit est fabriqué dans des conditions adéquates, qu'elle utilise toutes les informations fournies par les institutions existantes et qu'elle les stimule sans cesse pour qu'elles rendent ces informations plus précises et utilisables, de manière à fournir à l'acheteur individuel ces informations disponibles qui lui font pourtant si cruellement défaut, comme on l'a vu.

D'ailleurs, il se peut que les informations officielles déjà existantes aient été jusqu'ici si inefficaces précisément faute d'une telle société bénévole. Il s'est avéré vain de publier dans la presse que tels chocolats très à la mode actuellement sont faits par des enfants italiens dont les habitudes hygiéniques sont tellement exécrables que les médecins censés les visiter pour établir leur aptitude physique à travailler dans les ateliers ont exigé qu'ils se lavent, qu'ils changent leurs vêtements et qu'ils se coupent les cheveux avant de les examiner. Lesdits chocolats sont tout aussi populaires qu'avant cette révélation. Il s'est avéré vain de publier dans la presse que tel bouillon dont la publicité exalte si largement la qualité et les effets bénéfiques sur les malades, les personnes âgées et les petits enfants est préparé si près des réserves de produits fertilisants de l'établissement d'emballage que des inspecteurs d'usine ont fait un malaise pendant leur visite dans les bâtiments. Ce bouillon continue d'être servi pendant les repas de ceux qui ambitionnent d'améliorer leur position sociale. Il est vain de publier chaque année dans la presse que les sweaters et leurs victimes, après une "haute saison " pendant laquelle ils travaillent quatorze, dix-huit ou même vingt heures par jour, crèvent la faim de longues périodes durant, livrés entièrement à eux-mêmes; que la tuberculose, auparavant presque inconnue des Juifs russes, est maintenant appelée habituellement "la maladie du tailleur ", puisqu'elle est devenue une caractéristique typique des victimes des sweaters à cause des conditions inhumaines dans lesquelles ils travaillent. Les déclarations officielles sur tous ces sujets, paisiblement enterrées dans les rapports officiels, n'atteignent et n'influencent pas la masse des acheteurs. Qu'il soit dit en passant : il est vrai que la communauté tirerait un bénéfice d'autant plus grand d'une manière plus rigoureuse de faire respecter les ordonnances et les règles en coopérant à travers des associations bénévoles avec les agents publics chargés d'écrire ces rapports; car, en l'absence de preuves tangibles de l'existence d'une opinion publique éclairée et organisée, l'histoire des agents intègres chassés de leur poste, des agents corruptibles soudoyés, et des agents incompétents maintenus indéfiniment dans leurs fonctions constitue l'un des chapitres les plus sombres de l'histoire de l'industrie.

La Ligue Nationale des Consommateurs agit sur la base de la proposition suivante : que se constitue, afin de donner vie à une demande effective de biens produits dans des conditions justes, un nombre de consommateurs suffisamment élevé pour assurer des achats si importants qu'ils finiront par compenser les dépenses supplémentaires que subiront les employeurs dotés de sentiments humanistes.

27 Pour ce faire, la Ligue Nationale des Consommateurs a ouvert une permanence dans la ville de New York, et a entamé un travail systématique d'organisation d'autres ligues dans les États outres celles des États de New York, Pennsylvanie, Massachusetts et d'Illinois qui lui préexistaient et qui la constituent déjà.

La Ligue Nationale des Consommateurs entreprend dans le courant cette année une enquête sur un secteur industriel précisément défini et ce, afin d'expérimenter des moyens de déterminer le pouvoir des acheteurs lorsqu'ils s'organisent pour atteindre des buts définis. Aux opérateurs de ce secteur manufacturier - la production de sousvêtements féminins en mousseline blanche - la Ligue Nationale des Consommateurs 
propose d'utiliser son étiquette et ses critères de référence, et s'engage à rendre publiques d'une manière large et continue les conditions humaines qui existent dans les entreprises qu'elle reconnaît.

Les critères actuellement adoptés incluent : que tous les biens soient fabriqués par le fabriquant dans ses propres bâtiments ; que toutes les conditions fixées par les lois sur les usines de l'État soient respectées; qu'aucun enfant de moins de seize ans soit employé ; que le temps de travail ne soit pas excédentaire. Il est souhaitable que, dans un délai raisonnable, il devienne possible d'inclure une condition concernant le salaire minimum - les quatre conditions déjà mentionnées sont déjà respectées dans les meilleurs ateliers dans le secteur manufacturier qui nous occupe.

Depuis la sortie de l'activité manufacturière de l'espace domestique, la seule grande fonction des femmes dans l'industrie a été l'achat. Non seulement toute nourriture utilisée par les foyers, mais une large partie des meubles et des livres, ainsi que les vêtements des hommes, des femmes et des enfants, sont préparés en visant directement la vente à des acheteurs féminins. Il est par conséquent naturel que le premier effort pour éduquer la masse des acheteurs divers à propos du pouvoir de l'acheteur soit fait par les femmes, auprès des femmes, et au profit des femmes et des enfants. Un tel effort ayant réussi, dans certaines limites, au sein de cette sphère, il est maintenant en train d'être généralisé sans distinction d'âge et de sexe ; et la Ligue est en train de demander la coopération des institutions éducatives et des sociétés savantes.

31 Le premier effort dans notre pays a été le fait de deux dames, Mme Frederick Nathan et Mme Charles Russell Lowell, dans la ville de New York, en 1890. Elles choisirent deux magasins où le traitement des salariés semblait être particulièrement humain; et, en fixant comme critère les données concernant ces magasins, elles écrivirent à 1400 gérants de magasins de l'île de Manhattan en leur demandant s'ils souhaitaient organiser le travail dans leurs magasins suivant ces critères-là, et que leurs magasins figurent ainsi dans une liste blanche qu'elles auraient rédigée. Parmi les 1400 interpellés, deux seulement ont répondu favorablement, mais, de ces débuts si modestes, est issue la Liste Blanche actuelle de la Ligue des Consommateurs de la ville de New York, qui réunit presque quarante grands magasins. Car les deux dames réussirent à organiser leurs amis, à faire connaitre leur association naissante des vendeurs au détail, à diffuser leur Liste Blanche et les critères sur lesquels elle se basait; et à éduquer l'opinion publique concernant le pouvoir des acheteurs de déterminer les conditions du travail dans les magasins pour la vente au détail.

Les principes, l'objet et le critère actuels de la Ligue des Consommateurs de New York sont les suivants :

PRINCIPES : I. L'intérêt de la communauté implique que tous les travailleurs doivent recevoir, non pas un salaire minimum, mais un salaire adéquat. II La responsabilité pour les pires maux dont souffrent les salariés revient aux acheteurs qui continuent d'acheter dans les commerces les moins chers sans se soucier des conditions de ces prix si bas. III. Par conséquent, le devoir du consommateur consiste à découvrir à quelles conditions les marchandises achetées sont produites, et à insister afin que ces conditions restent au moins décentes et cohérentes avec une existence respectable en ce qui concerne les travailleurs. IV. Ce devoir revient tout particulièrement aux consommateurs en ce qui concerne les produits du travail féminin, car les salaires des femmes peuvent être réduits presque sans limites, sauf s'ils sont artificiellement 
conservés au niveau de la survie par des arrangements de la part des travailleurs ou des consommateurs.

OBJET: En reconnaissant que la majorité des employeurs sont pratiquement impuissants en ce qui concerne l'amélioration des conditions de travail, en termes de temps et de salaires, s'ils ne sont pas soutenus par l'opinion publique, par la loi, et par l'action des consommateurs, la Ligue des Consommateurs déclare que l'objet de son action est l'amélioration des conditions des femmes et des enfants employés dans la ville de New York, tout en contribuant à constituer une opinion publique susceptible de guider les consommateurs vers la reconnaissance de leurs responsabilités, ainsi qu'à travers d'autres méthodes.

CRITÈRES D'UNE ENTREPRISE JUSTE : Salaires. Une entreprise juste est une entreprise dans laquelle un salaire égal est donné pour un travail d'une valeur égale, sans différences entre les sexes. Où les salaires minimums représentent six dollars par semaine pour des travailleuses adultes et dotées d'expérience et descendent en dessous de huit dollars uniquement dans des cas exceptionnels, dans les départements où les femmes sont seules employées. Où les salaires sont payés chaque semaine. Où les amendes, lorsqu'elles sont imposées, sont versées dans une caisse en faveur des employés. Où les salaires minimums des cash-girls représentent deux dollars par semaine, avec les mêmes conditions en ce qui concerne les paiements hebdomadaires et les amendes. Heures. Une entreprise juste est une entreprise où le temps de travail est entre huit heures du matin et six heures du soir (avec trois quart d'heures pour la pause-déjeuner), et où une mi-journée de congé est accordée chaque semaine pendant au moins deux mois d'été. Où un congé payé non inférieur à une semaine est accordé pendant la période estivale. Où toute heure supplémentaire est rémunérée. Conditions sanitaires. Une entreprise juste est une entreprise où les pièces pour travailler, les salles à manger et les espaces privés sont séparés les uns des autres, et respectent entièrement les lois sanitaires existantes. Où la loi actuelle concernant les chaises pour les vendeuses est respectée, et l'usage de chaises est accordé. Autres conditions. Une entreprise juste est une entreprise où un comportement humain et attentionné envers les employés est la règle. Où la fidélité et l'ancienneté obtiennent le respect qui leur est dû. Où les enfants de moins de quatorze ans ne sont pas employés.

La Ligue des Consommateurs de la ville de New York, qui n'a affaire qu'aux magasins de l'île de Manhattan, n'a fait appel qu'à la conscience des acheteurs. En leur demandant de choisir les magasins inclus dans la Liste Blanche, elle a affirmé sa volonté d'encourager les employeurs se conduisant humainement à continuer de suivre leur chemin, et d'inciter les autres à les imiter.

La réussite de cet appel a encouragé la Ligue à élargir son champ d'intervention; et, par-là, à élargir aussi les objectifs de son appel. La Ligue Nationale des Consommateurs demande que les acheteurs, en insistant dans l'achat de produits dotés de son étiquette, opèrent une discrimination en faveur de ceux producteurs manufacturiers qui traitent leurs employés d'une façon humaine, autant que faire se peut dans les conditions imposées par le système de la concurrence ; et qu'ils le fassent tant au nom de l'intérêt des employés qu'en ayant en vue la promotion de ces formes de manufactures qui sont les plus saines pour la communauté tout entière, contre les conditions qui font de la diffusion des épidémies un risque constant et considérable.

L'appel est toujours, comme auparavant, au nom des employés, mais aussi au nom d'une instance constituante bien plus vaste - le public des acheteurs dans sa totalité. 
39 Car, de toute évidence, c'est aussi un devoir social de promouvoir ces formes de manufacture qui tendent à fournir des produits sains, fabriqués dans des conditions justes, plutôt que des sweatshops avec tous les dangers pour la famille auprès de qui le travail est fait, et pour l'acheteur qui peut acheter toutes les maladies auxquelles nous avons fait allusion, malgré l'assurance désinvolte du vendeur: "Toutes nos marchandises sont produites dans notre propre atelier ».

40 L'appel de la Ligue Nationale des Consommateurs promet d'être de plus en plus profitable pour ces employeurs qui se soucient de s'adresser à leurs employés en tant que gens se respectant eux-mêmes, employés dans des conditions raisonnables, et payés proportionnellement à la valeur de leur travail. Nombre de tels employeurs ont salué la Ligue avec une bienvenue cordiale. Le propriétaire d'un atelier, connu depuis quarante ans pour la sélection soigneuse de ses employés, inhabituellement intelligent et basé dans un environnement d'un charme rare, ayant été visité par un représentant de la Ligue Nationale des Consommateurs, a déclaré que le public n'avait pas l'air d'être intéressé par ces aspects de son atelier.

41 Les propriétaires de telles entreprises subissent constamment une pression très intense à cause de la concurrence d'autres industriels qui adoptent des critères moins exigeants. Ainsi, ils ont besoin du soutien que peut fournir un corps organisé d'acheteurs. Une démonstration pratique de cela peut être trouvée dans la proposition spontanée de la part de nombreux employeurs d'utiliser l'étiquette de la Ligue des Consommateurs et de se charger des frais d'impression et de l'attacher eux-mêmes au produit. Une autre démonstration est l'aide fournie par un industriel doté d'une grande expérience pour élaborer la forme du contrat à souscrire, ainsi que de nombreux projets graphiques pour l'étiquette, dont est issue la forme qui est actuellement utilisée.

$42 \mathrm{Au}$ fur et à mesure que les membres et l'influence de la Ligue augmentent, ce soutien moral et financier à l'égard des employeurs humanistes stimulera vraisemblablement l'esprit d'émulation chez d'autres qui ont jusqu'ici été guidés davantage par le désir de faire des économies que par le souci d'une bonne organisation de leurs entreprises. Tel a été du moins l'effet constaté dans la ville de New York, où les employeurs les plus éclairés ont été les premiers à se conformer aux demandes de la branche locale de la Ligue, tandis que d'autres qui hésitaient, parfois même pendant des années, ont fini par prendre les mesures requises.

43 En reconnaissant que son travail doit être un travail d'éducation et d'organisation, la Ligue des Consommateurs a recherché la coopération des grandes institutions éducatives. Les Départements d'économie des Universités de Harvard, Columbia, Pennsylvanie et du Wellesley College ont été activement impliqués dans le travail des Ligues de leurs États. Le Département de sociologie de l'Université de Chicago a apporté des contributions d'une très grande valeur, tant les étudiants que la Faculté ayant aidé par la langue et la plume à discuter les pouvoirs et les devoirs du consommateur.

L'American Academy of Political and Social Science a publié dans ses Annales les actes de la discussion autour des fondements théoriques du travail de la Ligue. L'American Association for the Advancement of Science et l'American Social Science Association ont traité ce même sujet lors de leurs réunions récentes, et il fera l'objet d'une séance de l'American Economic Association lors de son prochain meeting pendant les fêtes de Noël. L'Association of Collegiate Alumnae a étudié, lors de sa réunion en octobre dernier, les «Nouvelles pratiques économiques dans les Collèges membres de 
l'Association ", en attribuant une importance toute particulière à l'enseignement de la théorie de la consommation. La General Federation of Women's Clubs a consacré, lors de sa réunion bi-annuelle en juin 1899, sa séance principale à la discussion des principes et des buts de la Ligue des Consommateurs. Les Fédération des États des clubs féminins et des clubs individuels ont été invités à investir ce sujet, en l'inscrivant au programme de leurs réunions publiques. En général, le pouvoir et l'utilité de la Ligue des Consommateurs dépendra largement de l'intelligence et du travail actif des organisations locales, et du degré de coopération de la part du public que ces dernières auront réussi à susciter. Actuellement, la Ligue constate que les consommateurs, même lorsqu'ils sont désorganisés, ont le pouvoir de faire cesser la production de n'importe quelle marchandise en s'interdisant de l'acheter, et, symétriquement, de promouvoir la production d'autres marchandises en les demandant. Lorsqu'ils sont organisés, même sous une forme très partielle, les consommateurs peuvent décider, dans certaines limites, dans quelles conditions sont produits les biens qu'ils désirent. Toutefois, les consommateurs n'ont jamais fait cela d'une manière systématique et éclairée, en dehors des pratiques de l'achat coopératif et des limites imposées à l'adultération de la nourriture grâce à une législation dont les ressorts ont été justement les efforts des acheteurs.

Le pouvoir de l'acheteur, qui est virtuellement illimité, grandit pratiquement à mesure que les acheteurs s'organisent et s'éduquent, établissent une communication directe avec les producteurs, s'informent eux-mêmes d'une manière précise sur les conditions de la production et de la distribution, et deviennent ainsi capables d'imposer leur propre volonté au lieu de se soumettre aux séductions et aux impulsions venant de publicitaires cyniques.

Bref, et en résumant, le but de la Ligue Nationale des Consommateurs est d'organiser une demande effective de biens produits dans des conditions justes. Pour ce faire, elle envisage : 1) d'enquêter sur les conditions données de la production et de publier les résultats de ces enquêtes ; 2) de recommander auprès du public, à travers son étiquette, les biens dont elle aura constaté qu'ils sont produits dans des conditions satisfaisantes ; 3) de faire appel à la conscience de l'acheteur en s'opposant par-là aux publicitaires qui n'ont de cesse d'interpeller la crédulité et la cupidité du public ; 4) de coopérer avec, et d'encourager par tous les moyens légitimes, les employeurs dont le travail est accompli dans des conditions humaines et éclairées; 5) d'impulser des mesures législatives ultérieures pour la protection des acheteurs et des employés; 6) de coopérer avec ces officiers publics dont le devoir est d'enquêter sur les conditions de la production et de la distribution, ou d'appliquer des lois et des ordonnances ayant partie liée avec ces conditions ; 7) de constituer des organisations d'acheteurs poursuivant les objectifs susmentionnés.

\section{Recension de Poverty, par Robert Hunter, New York, The Macmillan Co., 1904 (in American Journal of Sociology, 01/01/1905, p. 555-556)}

Dans cet ouvrage, titré "Pauvreté », Robert Hunter a rendu aux Etats-Unis le même service que Frederick Engels a rendu à l'Angleterre il y a soixante ans en publiant son ouvrage sur La situation de la classe ouvrière. Dans l'un comme dans l'autre cas, l'auteur a révélé la lutte des pauvres qui vivent de leur salaire et de ceux dont les conditions se 
sont tellement dégradées qu'ils ont sombré plus bas encore que les salariés; et dans les deux cas l'ampleur et les dimensions du tableau dressé par l'auteur dépassent tout ce qui a été essayé auparavant dans un domaine similaire.

Le livre de Frederick Engels n'a pas été rendu obsolète par le travail d'enquêteurs plus récents. Il reste l'exposition classique de la vie des pauvres en Angleterre dans les années 1840 et 1850 . Certaines prophéties du jeune auteur ne se sont pas réalisées après soixante ans, car le don de lire l'avenir sans la moindre bévue n'a pas été accordé à l'homme. À partir de ce constat, Robert Hunter a lutté pour éviter les séductions de la prophétie et s'est borné à la description et à l'interprétation de phénomènes au milieu desquels nous vivons tous, bien qu'une minorité seulement possède le regard requis pour le travail de l'interprétation.

Nul homme qui se consacre à étudier la philanthropie ou la sociologie ne peut oser ignorer cet ouvrage. Ses positions générales concernant l'ampleur de la pauvreté et ses causes immédiates peuvent très facilement devenir un sujet pour de vastes et utiles discussions. Il est raisonnable de s'attendre à ce que le reproche d'exagérer soit adressé à l'auteur en ce qui concerne l'ampleur de la pauvreté telle qu'il la définit et aussi du point de vue du rapport à l'immigration. Mais des efforts honnêtes et sans préjugé pour réfuter la position de $\mathrm{M}$. Hunter ne pourront éviter de mettre une fois de plus en lumière la regrettable imprécision des figures officielles sur les publications desquelles les États et même le Gouvernement fédéral fondent des dépenses considérables dont les résultats sont affligeants d'incompétence scientifique.

La liste des autorités citées est peut-être affaiblie, des fois, par l'inclusion de noms aussi peu persuasifs aux yeux du lecteur scientifiquement informé que ceux de Mrs. Van Vorst et de Mr. W. J. Ghent ; mais lorsque toutes les déductions à partir du principe d'inclusion ont été tirées, l'ensemble des faits que cet ouvrage porte à notre attention doit imposer le respect de tout profane dépourvu de préjugés.

\section{Recension de A New Conscience and an Ancient Evil, par Jane Addams, Hull House, Chicago, Macmillan, 1912 (in American Journal of Sociology, 09/01/1912, p. 271-272)}

51 La qualité caractéristique de ce petit ouvrage consiste dans sa véridicité que l'espérance rend vivace et la connaissance éclaire. Il est complètement dépourvu de ce parfum malsain de scandale qui diminue la valeur de nombreux écrits récents sur le même sujet. Le livre se développe de manière organique à partir du contact direct de l'auteur avec des voisins dont les filles sont tout particulièrement exposées, de par leur pauvreté, aux ravages de cet ancien fléau.

La connaissance directe de la part de Miss Addams, depuis une vingtaine d'années, d'un quartier surpeuplé est enrichie par une militance active au sein de l'Association pour la Protection de la Jeunesse - une organisation qui n'a pas son pareil dans le monde quant à son champ d'activité et à son efficacité auprès des jeunes de la cité qui est son lieu d'exercice.

La thèse de l'auteur est que "plusieurs indices annoncent une nouvelle conscience, dont la force augmente en se développant dans plusieurs directions et à propos de 
laquelle nous pouvons sobrement espérer qu'elle finira par se dresser contre cet incroyable fléau social, malgré l'ancienneté de celui-ci ».

L'argumentation est annoncée par les titres suggestifs des six chapitres : « Une nouvelle conscience face à un fléau ancien »: Chapitre I - Déduite par analogie ; Chapitre II Suivant des actes juridiques récents; Chapitre III - Selon l'amélioration des conditions économiques ; Chapitre IV - Du point de vue de l'éducation morale et de la protection légale des enfants; Chapitre V - Du point de vue des associations philanthropiques d'aide et de protection; Chapitre VI - Du point de vue du contrôle social.

L'ouvrage ne contient pas de bibliographie, ni d'analyse de la littérature existante sur ce sujet, il ne fait aucune tentative pour traiter l'aspect quantitatif de ce fléau. C'est un ouvrage entièrement interprétatif et humain, et, à l'instar de toute l'œuvre de l'auteur, c'est un appel adressé aux forces sociales et éthiques de notre époque pour appliquer leur ardeur et leur énergie à la transition de notre chaos social à cette vie sociale noble et ordonnée qui inspire nos espoirs pour l'avenir.

\section{Recension de The Solution of the Child Labor Problem, par Scott Nearing, New York, Moffat, Yard \& Company, 1911 (in American Political Science Review, 02/01/1912, p. 145-146)}

Cet ouvrage petit et compact consacré à «La solution du problème du travail des enfants » constitue une contribution remuante à la recherche d'une telle solution. Le style étincelant de l'auteur, et la substance constructive de son livre, le distinguent positivement des platitudes arides des sympathisants sans connaissance et des experts sans style littéraire à cause desquels la cause des enfants ouvriers a beaucoup souffert ces dernières années.

Certaines propositions de l'auteur sont déjà une réalité ailleurs, par exemple l'éducation prolongée des jeunes garçons dans les écoles de Munich et dans d'autres villes allemandes. D'autres font l'objet d'une activité d'agitation incessante, guidée par Mrs Sidney Webb, de la part des soutiens du rapport minoritaire auprès de la Commission pour les lois anglaises sur la pauvreté, publié en 1909. Et encore d'autres mesures existent dans certains pays et font l'objet de l'intérêt du législateur chez nous. Ainsi, des bureaux pour le salaire minimum existent depuis 1893 en Australasie et en Angleterre depuis 1910. Des décrets concernant de tels bureaux ont été envisagés par les législateurs du Minnesota, du Wisconsin et du Massachusetts. Les partisans de telles mesures étaient à chaque fois aussi personnellement engagés dans le problème de la réforme du travail des enfants.

Un critique bienveillant pourrait être excusé de mettre en évidence une certaine tendance de l'auteur à donner des coups sur la mauvaise tête lorsque M. Nearing reproche aux réformateurs du travail des enfants les péchés d'omission et de commission des éducateurs. L'auteur de ces notes, engagé pendant un quart de siècle pour la réforme du travail des enfants, n'a jamais vécu ni constaté les jouissances auxquelles fait allusion la phrase suivante, non démontrée et indémontrable, de M. Nearing : 
Tandis que le réformateur jouit intensément à cause des statistiques attestant une diminution du travail des enfants, les victimes de cette diminution sont dans la nature, souffrent la faim ou les deux en même temps.

Dans les rares cas où des statistiques fiables existent concernant la diminution du travail des enfants, elles sont associées à une augmentation de la scolarisation dans les niveaux d'instruction prévus par la combinaison entre la loi sur l'école obligatoire et la loi sur le travail des enfants. Cela a été le cas depuis quelques années à Chicago et à New York. Il y a aussi des signes encourageants ces derniers mois dans l'État de M. Nearing, la Pennsylvanie.

\section{ABSTRACTS}

Traduction de textes consacrés à la construction des connaissances dans l'intervention sociale

\section{INDEX}

Geographical index: États-Unis

Mots-clés: consommateurs, travailleurs, condition du travail, enquêtes

Chronological index: Fin du XIXème siècle-début du XXème siècle

\section{AUTHORS}

\section{FLORENCE KELLEY}

Militante pour les droits des travailleurs et des femmes, traductrice de la Condition de la classe ouvrière en Angleterre et correspondante d'Engels 